\title{
VARIABILITY OF THE ULTRAVIOLET CONTINUUM AND EMISSION LINES OF NGC 3783
}

\author{
G.A. REICHERT \\ Universities Space Research Association \\ NASA-GSFC, Code 668, Greenbelt, MD 20771
}

On behalf of the International AGN Watch, I report on the results of intensive ultraviolet spectral monitoring of the Seyfert 1 galaxy NGC 3783. The nucleus of NGC 3783 was observed with the International Ultraviolet Explorer satellite on a regular basis for a total of seven months, once every 4 days for the first 172 days and once every other day for the final 50 days. Significant variability was observed in both continuum and emission-line fluxes. The light curves for the continuum fluxes exhibited two well-defined local minima or "dips," the first lasting $\lesssim 20$ days and the second $\lesssim 4$ days, with additional episodes of relatively rapid flickering of approximately the same amplitude. As in the case of NGC 5548 (the only other Seyfert galaxy that has been the subject of such an intensive, sustained monitoring effort), the largest continuum variations were seen at the shortest wavelengths, so that the continuum became "harder" when brighter. The variations in the continuum occurred simultaneously at all wavelengths $(\Delta t<2$ days). Generally, the amplitude of variability of the emission lines was lower than (or comparable to) that of the continuum. Apart from MgII (which varied little) and $\mathrm{N} \mathrm{v} \mathrm{(which} \mathrm{is} \mathrm{relatively} \mathrm{weak} \mathrm{and} \mathrm{badly} \mathrm{blended} \mathrm{with} \mathrm{Ly} \alpha$ ) the light curves of the emission lines are very similar to the continuum light curves, in each case with a small systematic delay or "lag." As for NGC 5548, the highest ionization lines seem to respond with shorter lags than the lower ionization lines. The lags found for NGC 3783 are considerably shorter than those obtained for NGC 5548, with values of (formally) $\sim 0$ days for He II $+\mathrm{O}$ III], and $\sim 4$ days for Ly $\alpha$, and Civ. The data further suggest lags of $\sim 4$ days for $\mathrm{Si}$ IV $+\mathrm{O}$ IV], and $8-30$ days for $\mathrm{Si} I I I]+\mathrm{CIII}]$. Uncertainties in these quantities are likely to be of order 2 3 days for the stronger features (Ly $\alpha, \mathrm{C}$ IV), and $3-4$ days for the weaker ones (He II + O III], Si IV+O IV], Si III] $+\mathrm{C} \mathrm{III]).} \mathrm{Mg} \mathrm{II} \mathrm{lagged} \mathrm{the} 1460 \AA$ continuum by $\sim 9$ days, although this result depends on the method of measuring the line flux, and may in fact be due to variability of the underlying Fe II lines. Correlation analysis further shows that the power density spectrum contains substantial unresolved power over time scales of $\lesssim 2$ days, and that the character of the continuum variability may change with time.

This research was supported by NASA grant NAG5-1824 to Ohio State University and NASA contract NAS5-32474 to Computer Sciences Corporation.

403

T. J.-L. Courvoisier and A. Blecha: Multi-Wavelength Continuum Emission of AGN, 403.

(C) 1994 IAU. Printed in the Netherlands. 